\title{
Optical vortex mode generation by nanoarrays with a tailored geometry
}

\author{
Mathew D. Williams, Matt M. Coles, David S. Bradshaw, David L. Andrews* \\ School of Chemistry, University of East Anglia, Norwich Research Park, Norwich NR4 7TJ, U. K.
}

\begin{abstract}
Light generated with orbital angular momentum, commonly known as an optical vortex, is widely achieved by modifying the phase structure of a conventional laser beam through the utilization of a suitable optical element. In recent research, a process has been introduced that can produce electromagnetic radiation with a helical wave-front directly from a source. The chirally driven optical emission originates from a hierarchy of tailored nanoscale chromophore arrays arranged with a specific propeller-like geometry and symmetry. In particular, a nanoarray composed of $n$ particles requires each component to be held in a configuration with a rotation and associated phase shift of $2 \pi / n$ radians with respect to its neighbor. Following initial electronic excitation, each such array is capable of supporting delocalized doubly degenerate excitons, whose azimuthal phase progression is responsible for the helical wave-front. Under identified conditions, the relaxation of the electronically-excited nanoarray produces structured light in a spontaneous manner. Nanoarrays of escalating order, i.e. those containing an increasing number of components, enable access to a set of topological charges of higher order. Practical considerations for the development of this technique are discussed, and potential new applications are identified.
\end{abstract}

Keywords: optical angular momentum, Laguerre-Gaussian, optical vortices, quantum optics, chirality, structured light

\section{INTRODUCTION}

Structured light beams with a twisted wave-front, which accommodate a distinctive phase variation and topology in the electromagnetic fields, and an associated orbital angular momentum, ${ }^{1}$ allow for numerous novel applications. These include: micro-particle rotary manipulation, ${ }^{2}$ the entanglement of optical states, ${ }^{3-4}$ the transmission of quantum information, ${ }^{5}$ and the use of associated optical elements for image enhancement. ${ }^{6-7}$ While the fundamental properties of such 'optical vortex' beams are now well-known, this field continues to generate further discoveries: it also invites reconsideration of the amount of information that can be conveyed or measured within an individual photon. ${ }^{8}$ In a beam with a topological charge $l$ (an integer signifying the number of wavelengths within which each part of the wavefront surface twists once about the propagation axis) individual photons convey an orbital angular momentum $l \hbar$, in addition to the more commonly recognized spin angular momentum that connects to a polarization state.

The formation of vortex radiation usually arises via the conversion of a conventional Gaussian beam with spatial light modulators, ${ }^{9-10}$ a $q$-plate ${ }^{11}$ or a spiral phase plate. ${ }^{12}$ Such procedures often create an optical output containing more than one vortex mode, with positive and negative topological charges separable through angular filtering. ${ }^{13-14}$ Previously, it was considered inconceivable for optical vortex radiation to be produced directly with a specific topological charge. To achieve such spontaneous emission requires correlation between the spatial and electronic properties of the emitters, implausible with the employment of atomic components within a conventional source. However, it has now been demonstrated that the specific conditions for direct generation of vortex radiation can be satisfied in molecular arrays of specified geometry and symmetry. ${ }^{15}$ Furthermore, the tailored design of these arrays provides for generating vortices of arbitrary topological charge. In Section 2 the emitter arrays, and their associated symmetries, are introduced and Section 3 presents contour simulations for the distribution of azimuthal phase. The paper concludes with a general discussion.

Complex Light and Optical Forces VIII, edited by David L. Andrews, Enrique J. Galvez, Jesper Glückstad, Proc. of SPIE Vol. 8999, 89990Q - (c) 2014 SPIE · CCC code: 0277-786X/14/\$18 - doi: 10.1117/12.2036772 


\section{MOLECULAR ARRAY AND SYMMETRY}

Consider a molecular array containing $n$ units of identical composition, ${ }^{16}$ each with its own electronic integrity, rigidly held by a host material so that Coulombic interactions are possible, but significant orbital overlap does not occur. Each unit is assumed to act as a chromophore, i.e. it displays characteristic absorption and emission processes in the visible wavelength region. In synthetic materials, the chromophores will in many cases be sub-units of a supramolecular structure acting as a support and a dielectric insulator between the individual components. While the wavefunction of the electronic ground state for such an array essentially includes a product of the ground state wavefunctions of each individual unit, the corresponding excited states involves delocalization and a field splitting is experienced, associated with the lowering of symmetry due to nearest-neighbor couplings.

To achieve the necessary array orientations for the emission dipoles, a structure can be envisaged as follows. Consider a cylinder represented as a series of connectors, all equidistantly dispersed, drawn perpendicular to the perimeters of two congruent circles displaced along the axis. ${ }^{17}$ If the base of the cylinder is rotated relative to the top, the connectors adopt a slanted configuration; taking a cross-section of this construct provides the essence of the dipole orientation required in the following, as illustrated in Figure 1. We focus on the general case of three or more $(n)$ such emitters, centered upon a rotational symmetry axis of order $n \cdot{ }^{18}$ [Two nanoemitters may generate a planar phase discontinuity, but vortex emission is not possible for such a case. $]^{19}$ This configuration confers precisely equivalent electronic coupling between each adjacent pair, on the assumption that there is negligible interaction with potentially less highly symmetric surroundings. The Schoenflies point group symmetry designation of such a system is $C_{n}$ or $C_{n h},{ }^{20}$ depending on the absence or presence, respectively, of reflection symmetry in the plane that includes the centers of each component of the array. For simplicity the former, more general, case (signifying 3D, rather than planar, chirality) is adopted; this removes a restriction for the chromophore emission dipoles to lie in the plane.

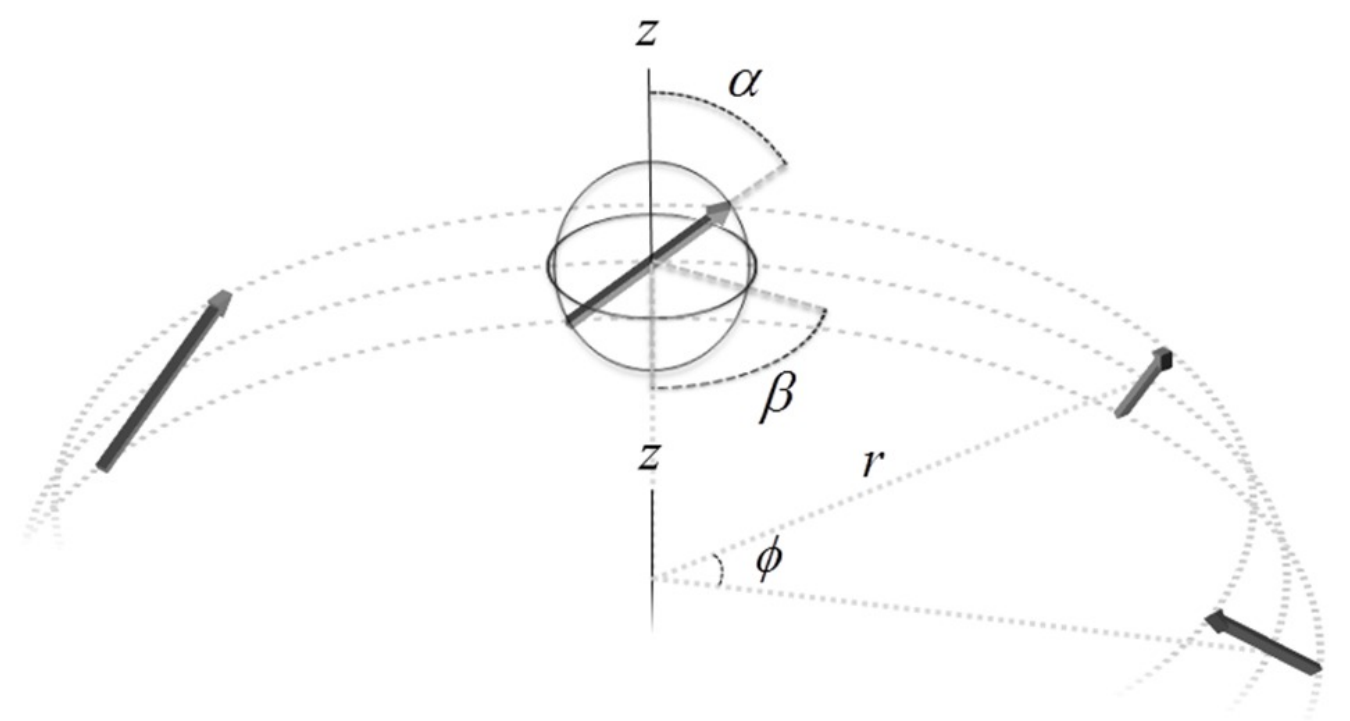

Figure 1. Nanoarray comprising of $n$ dipoles with ring placement represented by $(r, \theta, z)$ and orientation $(\alpha, \beta, z)$.

To find the electronic excited states supported by such an array, the first step is to block diagonalize the Hamiltonian operator, which can be cast in the following matrix representation (rows and columns denoting individual chromophore components):

$$
H_{r s}=E_{u} \delta_{r s}+\left(\boldsymbol{\mu}_{r} \cdot \mathbf{V}\left(\mathbf{R}_{r s}\right) \cdot \boldsymbol{\mu}_{s}\right)\left(\delta_{r-1, s(\bmod n)}+\delta_{r(\bmod n), s-1}\right)
$$


where $\mu_{r}$ is the electric dipole operator for component $r$ and the second term in the equation denotes electrodynamic coupling between dipoles. It emerges that the coupling between adjacent dipoles produces a displacement of the energy eigenvalues from their unperturbed values, $E_{u}$, now taking the form $E_{u}+2 U \cos (2 \pi q / n)$ with $1 \leq q \leq\lfloor(n-1) / 2\rfloor$ and

$$
\begin{aligned}
U & \equiv V_{r,(r+1) \bmod n}\left(k_{u}, \mathbf{R}_{r,(r+1) \bmod n}\right) \equiv V_{12}\left(k_{u}, \mathbf{R}_{12}\right) \\
& =\frac{e^{\mathrm{i} k_{u} R}}{4 \pi \varepsilon_{0} R^{3}}\left[\left\{1-\mathrm{i} k_{u} R-\left(k_{u} R\right)^{2}\right\}\left(\boldsymbol{\mu}_{1}^{0 u} \cdot \boldsymbol{\mu}_{2}^{0 u}\right)-\left\{3-3 \mathrm{i} k_{u} R-\left(k_{u} R\right)^{2}\right\}\left\{\left(\boldsymbol{\mu}_{1}^{0 u} \cdot \hat{\mathbf{R}}_{12}\right)\left(\boldsymbol{\mu}_{2}^{0 u} \cdot \hat{\mathbf{R}}_{12}\right)\right\}\right] .
\end{aligned}
$$

This expression shows that each nearest-neighbor interaction produces a coupling of identical magnitude to that existing between chromophores 1 and 2, as befits the ring symmetry. In equation (2), the nearest neighbor distance $R$ is defined by $\mathbf{R}_{1}-\mathbf{R}_{2} \equiv \mathbf{R}_{12}=R \hat{\mathbf{R}}_{12}$, the carat here denoting a unit vector; the individual electric dipole transition moments are defined by $\boldsymbol{\mu}_{r}^{0 u} \equiv\left\langle\xi^{r ; 0}\left|\boldsymbol{\mu}^{(r)}\right| \xi^{r ; u}\right\rangle$ and $k_{u}=E_{u} / \hbar c$.

Diagonalizing the system Hamiltonian elicits wavefunctions for excited states in which the electronic energy is substantially delocalized around the ring; in standard terminology these are referred to as excitons. Generally, such excitons emerge in various symmetry forms, with each distinct symmetry associated to a particular energy. Our focus will be on the wavefunctions for these specific forms of exciton, to be indexed with a numerical integer $p$. The corresponding quantum states, signifying the lowest electronically excited levels of the array, comprise superpositions of $n$ basis states in each of which a single chromophore is excited and the others reside in their ground state. In Dirac notation, the exciton state vectors are thus expressible as:

$$
\left|\psi_{p}\right\rangle=\frac{1}{\sqrt{n}} \sum_{r=1}^{n} \varepsilon_{n}^{(r-1) p}\left|\xi^{r ; u}\right\rangle \prod_{s \neq r}\left|\xi^{s ; 0}\right\rangle, p \in\{1, \ldots, n\}
$$

Here $\left|\xi^{r ; u}\right\rangle$ is the state vector for chromophore $r$ in its excited state $u,\left|\xi^{r ; 0}\right\rangle$ represents the corresponding ground state, and $\varepsilon_{n}=\exp (2 \pi i / n)$. The number of exciton states equals the order of the symmetry point group, again simply $n$ in the case of $C_{n}$ symmetry. These states now fall into two distinct symmetry categories: there are $\lfloor(n-1) / 2\rfloor$ (the largest integer not greater than $(n-1) / 2$ ) doubly degenerate pairs with Schoenflies irreducible representations conventionally labeled $E_{q}$, with $1 \leq q \leq\lfloor(n-1) / 2\rfloor$; also there is one totally symmetric, non-degenerate state with symmetry label $A$. Furthermore, in the case of even-valued $n$, there is an additional non-degenerate state of $B$ symmetry. The $A$ states arising from $p=n$ do not exhibit a phase progression around the ring, since in Eq. (3) the factor $\varepsilon_{n}^{(r-1) p}$ then equals unity. Equally the phase progression of $B$ states, where $p=n / 2$, lacks specific circularity. Attention is therefore directed to the doubly degenerate $E_{q}$ states, each component of which displays a circular phase progression of equal topological charge $q$, but with opposite signs. For example, in the $E_{4}$ exciton state of a nine-member ring comprising chromophores $r=\{1,2, \ldots, 9\}$, each electronically excited chromophore carries a phase factor of $\exp ( \pm 8 \pi \mathrm{ir} / 9)$, consistent with topological charges \pm 4 .

In consequence of the $q$-dependence of the energy levels, it is possible to selectively excite an individual $E_{q}$ state with a topological charge of specific magnitude. To identify the optical field produced by the ensuing exciton decay requires summing the retarded electric field components associated with each emitter, ${ }^{21}$ accounting for their relative phases as given by Eq. (3). The result is as follows:

$$
\mathbf{E}_{p}\left(\mathbf{R}_{D}\right)=\sum_{r}^{n} \frac{e^{i k R_{D r}} \varepsilon_{n}^{(r-1) p}}{4 \pi \varepsilon_{0} R_{D r}^{3}}\left\{\left[\left(\hat{\mathbf{R}}_{D r} \times \boldsymbol{\mu}_{r}^{0 u}\right) \times \hat{\mathbf{R}}_{D r}\right] k^{2} R_{D r}^{2}+\left[3 \hat{\mathbf{R}}_{D r}\left(\hat{\mathbf{R}}_{D r} \cdot \boldsymbol{\mu}_{r}^{0 u}\right)-\boldsymbol{\mu}_{r}^{0 u}\right]\left(1-i k R_{D r}\right)\right\},
$$

expressing the field detected at a position $\mathbf{R}_{D}$. From this result we can identify the phase distribution of the associated scalar optical field, $\theta_{i}(\mathbf{R})=\arg \left\{E_{p ; i}\left(\mathbf{R}_{D}\right)\right\}$, where the individual Cartesian components, indexed by $i$, are explicitly exhibited in the following section for the case of a nine-particle ring. 


\section{CONTOUR MAPS OF THE PHASE}

For a nine-emitter configuration, we consider the individual chromophores to be equidistantly distributed around the perimeter of a ring of radius $\lambda / 150 \pi$ (where $\lambda$ is the normal emission wavelength of an isolated chromophore), each suitably oriented such that the whole conforms to the $C_{9}$ point group. Specifically, for the conditions used in the calculations leading to the displayed results, the angle between the normal to the plane and the radial position vector of the corresponding emitter is taken as $\pi / 4$, as is the angle subtended by each emission dipole moment onto the plane containing the array centers. Moreover, the coupling constant $U$ is assumed to be positive.

In Fig. (2) each color represents a unique electromagnetic phase associated with the corresponding optical vortex emission is represented for a selection of orbital angular momenta. The contours radiating in a spiral manner from the ring of emitters at the center are lines of constant phase. The phase clearly cycles through $2 \pi^{\mathrm{c}}$ around concentric circles of constant radius from the ring center. A field singularity is located along the central axis; it is also notable that the internal structure of phase (within the ring) identically opposes the phase at the corresponding location immediately external to the ring.
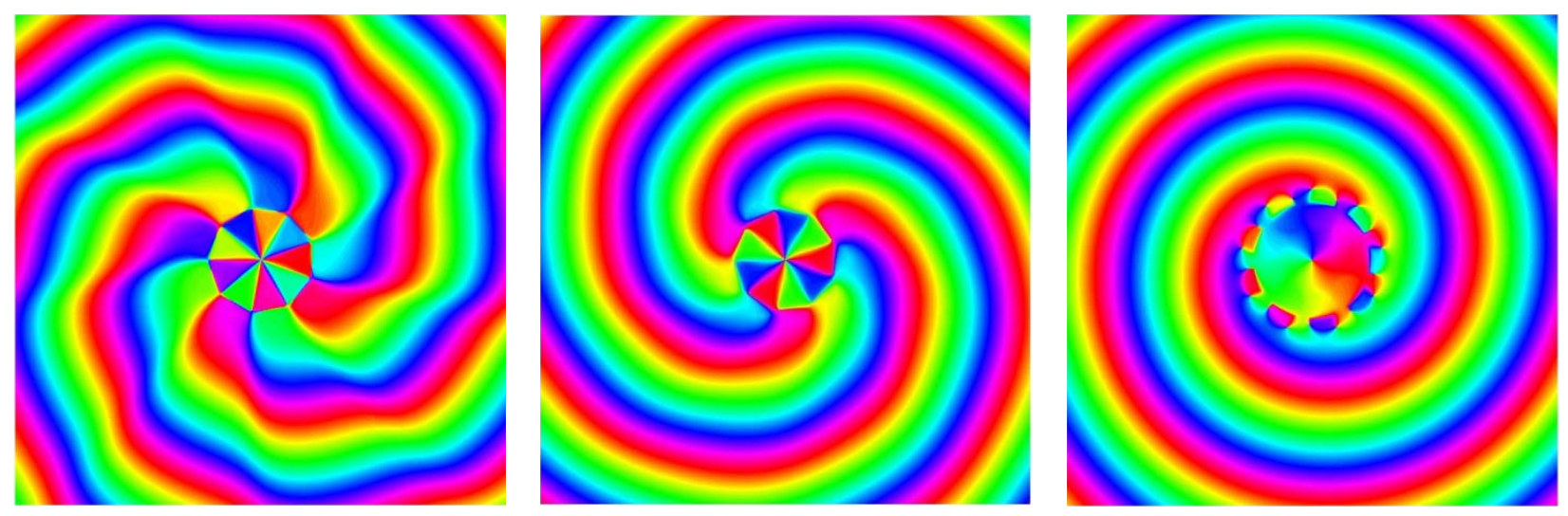

Figure 2. Electromagnetic phase of optical vortices emitted by an array comprising nine chromophores, equidistantly distributed around a ring's perimeter of radius $\lambda / 150 \pi$. The orbital angular momentum values (left to right) are $l=4$, $l=-3$ and $l=1$.

\section{DISCUSSION}

Our results suggest a need to rethink the practicality of directly generating vortex light, which was not envisaged in some of the earlier literature. ${ }^{22}$ Now it proves possible to conceive structures that, as a whole, are capable of supporting extensively delocalized states whose electronic relaxation emits photons with a topological charge and an associated orbital angular momentum. For practical applications, one potential advantage to using such a scheme might be a reduction in device size, afforded by the opportunity to directly form the sought emission as opposed to having to modify the output of another source. ${ }^{23}$ The possible impact in technological circles is further bolstered by the more recent rapid advancements in the sorting of OAM endowed beams. ${ }^{24-25}$ A range of prospective informatics applications emerges.

\section{ACKNOWLEDGMENTS}

The authors would like to thank the University of East Anglia, EPSRC and Leverhulme Trust for funding this research. 


\section{REFERENCES}

[1] Allen, L., Beijersbergen, M. W., Spreeuw, R. J. C. and Woerdman, J. P., "Orbital angular momentum of light and the transformation of Laguerre-Gaussian laser modes,” Phys. Rev. A 45, 8185-8189 (1992).

[2] Simpson, N. B., Dholakia, K., Allen, L. and Padgett, M. J., "Mechanical equivalence of spin and orbital angular momentum of light: An optical spanner," Opt. Lett. 22, 52-54 (1997).

[3] Mair, A., Vaziri, A., Weihs, G. and Zeilinger, A., "Entanglement of the orbital angular momentum states of photons," Nature 412, 313-316 (2001).

[4] Romero, J., Giovannini, D., Franke-Arnold, S., Barnett, S. M. and Padgett, M. J., "Increasing the dimension in high-dimensional two-photon orbital angular momentum entanglement,” Phys. Rev. A 86, 012334 (2012).

[5] Gibson, G., Courtial, J., Padgett, M., Vasnetsov, M., Pas'ko, V., Barnett, S. and Franke-Arnold, S., "Free-space information transfer using light beams carrying orbital angular momentum,” Opt. Express 12, 5448-5456 (2004).

[6] Furhapter, S., Jesacher, A., Bernet, S. and Ritsch-Marte, M., "Spiral phase contrast imaging in microscopy,” Opt. Express 13, 689-694 (2005).

[7] Jack, B., Leach, J., Romero, J., Franke-Arnold, S., Ritsch-Marte, M., Barnett, S. M. and Padgett, M. J., "Holographic Ghost Imaging and the Violation of a Bell Inequality,” Phys. Rev. Lett. 103, 083602 (2009).

[8] Wang, J., Yang, J.-Y., Fazal, I. M., Ahmed, N., Yan, Y., Huang, H., Ren, Y., Yue, Y., Dolinar, S., Tur, M. and Willner, A. E., "Terabit free-space data transmission employing orbital angular momentum multiplexing," Nat. Photonics 6, 488-496 (2012).

[9] Heckenberg, N. R., Mcduff, R., Smith, C. P. and White, A. G., "Generation of Optical-Phase Singularities by Computer-Generated Holograms,” Opt. Lett. 17, 221-223 (1992).

[10] Ostrovsky, A. S., Rickenstorff-Parrao, C. and Arrizón, V., "Generation of the perfect optical vortex using a liquidcrystal spatial light modulator,” Opt. Lett. 38, 534-536 (2013).

[11] Marrucci, L., Manzo, C. and Paparo, D., "Optical spin-to-orbital angular momentum conversion in inhomogeneous anisotropic media,” Phys. Rev. Lett. 96, 163905 (2006).

[12] Beijersbergen, M. W., Coerwinkel, R. P. C., Kristensen, M. and Woerdman, J. P., "Helical-Wave-Front LaserBeams Produced with a Spiral Phaseplate,” Opt. Commun. 112, 321-327 (1994).

[13] Berkhout, G. C., Lavery, M. P., Courtial, J., Beijersbergen, M. W. and Padgett, M. J., "Efficient sorting of orbital angular momentum states of light,” Phys. Rev. Lett. 105, 153601 (2010).

[14] O'Sullivan, M. N., Mirhosseini, M., Malik, M. and Boyd, R. W., "Near-perfect sorting of orbital angular momentum and angular position states of light,” Opt. Express 20, 24444-24449 (2012).

[15] Andrews, D. L., Coles, M. M., Williams, M. D. and Bradshaw, D. S., "Expanded horizons for generating and exploring optical angular momentum in vortex structures,” Proc. SPIE 8813, 88130Y-88130Y-13 (2013).

[16] Williams, M. D., Coles, M. M., Saadi, K., Bradshaw, D. S. and Andrews, D. L., "Optical Vortex Generation from Molecular Chromophore Arrays,” Phys. Rev. Lett. 111, 153603 (2013).

[17] Weisstein, E. W., [CRC Concise Encyclopedia of Mathematics], Chapman \& Hall/CRC, Boca Raton (2003). 
[18] Coles, M. M., Williams, M. D., Saadi, K., Bradshaw, D. S. and Andrews, D. L., “Chiral nanoemitter array: A launchpad for optical vortices,” Laser \& Photon. Rev. 7, 1088-1092 (2013).

[19] Ford, J. S., Bradshaw, D. S. and Andrew, D. L., "Signatures of exciton coupling in paired nanoemitters,” J. Phys. Chem. C 117, 12393-12396 (2013).

[20] Salthouse, J. A., Ware, M. J. and Ware, M. J., [Point Group Character Tables and Related Data], Cambridge University Press, London (1972).

[21] Andrews, D. L. and Bradshaw, D. S., "Virtual photons, dipole fields and energy transfer: a quantum electrodynamical approach,” Eur. J. Phys. 25, 845-858 (2004).

[22] Dávila Romero, L. C., Andrews, D. L. and Babiker, M., “A quantum electrodynamics framework for the nonlinear optics of twisted beams,” J. Opt. B: Quantum Semiclass. Opt. 4, 66 (2002).

[23] Mirhosseini, M., Magana-Loaiza, O. S., Chen, C., Rodenburg, B., Malik, M. and Boyd, R. W., "Rapid generation of light beams carrying orbital angular momentum,” Opt. Express 21, 30196-30203 (2013).

[24] Lavery, M. P., Roberston, D., Malik, M., Robenburg, B., Courtial, J., Boyd, R. W. and Padgett, M. J., “The efficient sorting of light's orbital angular momentum for optical communications,” Proc. SPIE 8542, 85421R (2012).

[25] Mirhosseini, M., Malik, M., Shi, Z. and Boyd, R., "Efficient separation of the orbital angular momentum eigenstates of light,” Nat. Commun. 4, 2781 (2013).

*david.andrews@physics.org 